\title{
Distribuição geográfica e hospedeiros quirópteros (Mammalia, Chiroptera) de moscas nicteribidas americanas (Diptera, Nycteribiidae) ${ }^{1}$
}

Gustavo Graciolli ${ }^{2}$

\begin{abstract}
Geographic distribution and bat hosts (Mammalia, Chiroptera) of the American nicteribiid batflies (Diptera: Nycteribiidae). The list of the 49 American nicteribiid batflies species is presented, with informations about countries and hosts that were found in the literature.

KEY WORDS. Nycteribiidae, Basilia, Hershkovitzia, hosts, geographic distribution
\end{abstract}

Este trabalho deve como objetivo apresentar dados sobre a distribuição geográfica de moscas nicteribidas americanas, bem como seus respectivos hospedeiros quirópteros, tendo em vista sintetizar as informações até o presente conhecidas.

A partir de GUIMARÃES \& D'ANDRETTA (1956) que resumiram e retificaram as informações disponíveis sobre distribuição e hospedeiros dos nicteribidas americanos, outros registros referentes à material encontrado no Canadá, e anteriormente não citados por aqueles autores são aqui incluídos. Também não foram considerados registros relativos a hospedeiros não-quirópteros, por claramente tratarem-se de equívocos na identificação do hospedeiro ou da procedência do parasito. Com exceção dos registros citados por GUIMARÃES \& D'ANDRETTA (1956), a citação repetida do mesmo material, por dois ou mais autores, não é aqui considerada.

As 49 espécies de nicteribidas estão citadas em ordem alfabética, assim como os países e seus respectivos hospedeiros. Ao final do trabalho, é apresentado um quadro resumindo contendo as ocorrências de nicteribidas por países (Tab. I).

Em relação aos hospedeiros, os seus nomes subespecíficos não foram utilizados; os nomes válidos e as sinonímias seguem Koopman (1993). Apenas uma exceção a de Phyllostoma sp., foi considerada devido ao fato de um grande número de espécies de morcegos filostomídeos ter sido indiscriminadamente alocado neste gênero no início do século (WENZEL et al. 1966).

1) Contribuição número 1270 do Departamento de Zoologia, Universidade Federal do Paraná.

2) Departamento de Zoologia, Universidade Federal do Paraná. Caixa Postal 19020, 81531-990 Curitiba, Paraná, Brasil. E-mail: mingau@bio.ufpr.br 
Basilia Miranda Ribeiro, 1903

\section{Basilia anceps Guimarães \& D’Andretta, 1956}

COLÔMBIA

Myotis nigricans (Schinz, 1821) (GUIMARÃES \& D'ANDRETTA 1956: 113). PANAMÁ

Myotis nigricans (Schinz, 1821) (GUIMARÃES 1966: 400).

Myotis simus Thomas, 1901 (GUIMARÃES 1966: 400). PERU

Myotis nigricans (Schinz, 1821) (GUIMARÃES \& D'ANDRETTA 1956: 113). VENEZUELA

Myotis nigricans (Schinz, 1821) (GUERRERO 1996: 672).

Myotis riparius Handley, 1960 (GUERRERo 1996: 672).

\section{Basilia andersoni Peterson \& Maa, 1970}

BRASIL

Histiotus velatus (I. Geoffroy, 1824) (PETERSON \& MAA 1970a: 1484).

URUGUAI

Eptesicus brasiliensis (Desmarest, 1819) (PETERSON \& MAA 1970a: 1484). Myotis nigricans (Schinz, 1821) (PETERSON \& MAA 1970a: 1484).

\section{Basilia anomala Guimarães \& D'Andretta, 1956}

GuATEMALA

Rhogeessa tumida H. Allen, 1866 (GUIMARÃES \& D'ANDRETTA 1956: 67). MÉXICO

Rhogeessa tumida H. Allen, 1866 (GUIMARÃEs \& D'ANDRETTA 1956: 67). NICARÁGUA

Myotis nigricans (Schinz, 1821) (GUIMARÃES 1972: 2).

VENEZUELA

Rhogeessa tumida H. Allen, 1866 (GUIMARÃES 1972: 1; 1977: 221).

\section{Basilia antrozoi (Townsend, 1893)}

CANADÁ

Antrozus pallidus (Le Conte, 1856) (CHILTON et al. 2000: 192).

ESTADOS UNIDOS

Antrozus pallidus (Le Conte, 1856) (GUIMARÃES \& D'ANDRETTA 1956:

117, 118; PETERSON 1960: 32, 33; 1963: 93; BRADSHAW \& ROSS 1961:

111; HANSEN 1964: 79; THEODOR 1967: 256; RICHERSON et al. 1992: 12; LEWIS 1996: 339).

Antrozus sp. (GUIMARÃes \& D'ANDRETTA 1956: 118; PETERSON 1960: 32). Plecotus rafinesquii Lesson, 1827 (GUIMARÃEs \& D'ANDRETTA 1956: 118). Tadarida brasiliensis (I. Geoffroy, 1824) (GUIMARÃES \& D'ANDRETTA 1956: 118). 


\section{MÉXICO}

Antrozus pallidus (Le Conte, 1856) (GUIMARÃES \& D'ANDRETTA 1956: 118). Leptonycteris nivalis (Saussure, 1860) (GUIMARÃES \& D'ANDRETTA 1956: 118).

Myotis californicus (Audubon \& Bachman, 1842) (GUIMARÃES \& D'ANDRETTA 1956: 118).

\section{Basilia astochia Peterson \& Maa, 1970}

COLÔMBIA

Platyrrhinus helleri (Peters, 1866) (PETERSON \& MAA 1970b: 1522).

Basilia bellardii (Rondani, 1878)

MÉXICO

Hospedeiro indeterminado (GUIMARÃES \& D'ANDRETTA 1956: 56, 57).

\section{Basilia bequaerti Guimarães \& D'Andretta, 1956}

COLÔMBIA

Micronycteris megalotis (Gray, 1842) (GUIMARÃES \& D'ANDRETTA 1956:

37).

PARAGUAI

Eptesicus furinalis (d'Orbigny, 1847) (GUIMARÃES \& D'ANDRETTA 1956: 37).

VENEZUELA

Hospedeiro indeterminado (GUIMARÃES \& D'ANDRETTA 1956: 37).

LOCALIDADE INDETERMINADA

Hospedeiro indeterminado (HURKA 1964: 77).

Basilia boardmani Rozeboom, 1934

ESTADOS UNIDOS

Myotis austroriparius (Rhoads, 1897) (GUIMARÃES \& D'ANDRETTA 1956: 47; RICE 1957: 30; HOBSON 2000: 291).

Myotis lucifugus (Le Conte, 1831) (PETERSON 1960: 33).

Hospedeiro indeterminado (GUIMARÃES \& D'ANDRETTA 1956: 47).

\section{Basilia carteri Scott, 1936}

ARGENTINA

Myotis albescens (E. Geoffroy, 1806) (AUTINO et al. 1999: 141; ClAPS et al. 1992: 88).

Myotis keaysi Allen, 1914 (AUTINO et al. 2000: 110).

Myotis nigricans (Schinz, 1821) (GUIMARÃES \& D'ANDRETTA 1956: 98).

Myotis riparius Handley, 1960 (AUTINo et al. 1999: 141; 2000: 110).

Myotis sp. (GUIMARÃES \& D'ANDRETTA 1956: 99).

Tadarida brasiliensis (I. Geoffroy, 1824) (GUIMARÃES \& D'ANDRETTA 1956: 99). 
BOLÍVIA

Molossus molossus (Pallas, 1766) (GUIMARÃES \& D’ANDRETTA 1956: 99).

Myotis nigricans (Schinz, 1821) (GUIMARÃES \& D'ANDRETTA 1956: 99;

THEODOR 1967: 273).

BRASIL

Eptesicus brasiliensis (Desmarest, 1819) (GRACIOLli \& CARVALHO 2001: 37).

Myotis albescens (E. Geoffroy, 1806) (THEODOR 1967: 273).

Myotis nigricans (Schinz, 1821) (GUIMARÃEs \& D'ANDRETTA 1956: 99).

Myotis sp. (GUIMARÃEs \& D'ANDRETTA 1956: 99).

Hospedeiro indeterminado (GUIMARÃES \& D'ANDRETTA 1956: 99).

PARAGUAI

Eptesicus furinalis (d'Orbigny, 1847) (GUIMARÃES \& D'ANDRETTA 1956: 98).

Molossops temminckii (Burmeister, 1854) (GUIMARÃES \& D’ANDRETTA 1956: 98).

Myotis albescens (E. Geoffroy, 1806) (GUIMARÃES \& D’ANDRETTA 1956: 98).

Hospedeiro indeterminado (GUIMARÃEs \& D'ANDRETTA 1956: 99).

URUGUAI

Myotis albescens (E. Geoffroy, 1806) (CLAPS et al. 1998: 113).

\section{Basilia constricta Guimarães \& D'Andretta, 1956}

COLÔMBIA

Eptesicus brasiliensis (Desmarest, 1819) (GUIMARÃES \& D’ANDRETTA 1956: 80).

\section{EQUADOR}

Myotis albescens (E. Geoffroy, 1806) (GUIMARÃES \& D’ANDRETTA 1956: 80).

Myotis nigricans (Schinz, 1821) (GUIMARÃES \& D’ANDRETTA 1956: 80).

Tonatia silvicola (d'Orbigny, 1836) (GUIMARÃES \& D'ANDRETTA 1956: 80). PERU

Hospedeiro indeterminado (GUIMARÃES \& D'ANDRETTA 1956: 80).

Myotis nigricans (Schinz, 1821) (GUIMARÃES \& D'ANDRETTA 1956: 80).

Uroderma bilobatum Peters, 1866 (GUIMARÃES \& D'ANDRETTA 1956: 80). VENEZUELA

Macrophyllum macrophyllum (Schinz, 1821) (GUIMARÃES \& D'ANDRETTA 1956: 80).

Hospedeiro indeterminado (GUIMARÃES \& D'ANDRETTA 1956: 80).

\section{Basilia corynorhini (Ferris, 1916)}

\section{ESTADOS UNIDOS}

Antrozus pallidus (Le Conte, 1856) (GUIMARÃES \& D’ANDRETTA 1956: 48).

Leptonycteris nivalis (WHITAKER JR. \& EASTERLA 1975: 248). 
Pipistrellus hesperus (H. Allen, 1864) (PETERSON 1960: 33).

Plecotus townsendi Cooper, 1837 (GUIMARÃES \& D'ANDRETTA 1956: 61;

BRADSHAW \& ROSS 1961: 111; THEODOR 1967: 266).

Plecotus rafinesquii Lesson, 1827 (PETERSON 1960: 33).

Plecotus sp. (PETERSON 1963: 93).

\section{Basilia costaricensis Guimarães \& D'Andretta, 1956}

Costa RicA

Hospedeiro indeterminado (GUIMARÃES \& D'ANDRETTA 1956: 106).

\section{Basilia cubana Hurka, 1970}

CUBA

Eptesicus fuscus (Beauvois, 1796) (HURKA 1970: 337).

Hospedeiro indeterminado (HURKA 1970: 337).

\section{Basilia currani Guimarães, 1943}

ARGENTINA

Myotis chiloensis (Tomes, 1857) (GUIMARÃES \& D'ANDRETTA 1956: 92). Myotis levis (I. Geoffroy, 1824) (AUTINo et al. 2000: 110).

Tadarida brasiliensis (I. Geoffroy, 1824) (GUIMARÃES \& D'ANDRETTA 1956: 92).

BRASIL

Eptesicus brasiliensis (Desmarest, 1819) (GUIMARÃES \& D'ANDRETTA 1956: 92).

Myotis albescens (E. Geoffroy, 1806) (GUTMARÃES \& D'ANDRETTA 1956: 92). Myotis ruber (E. Geoffroy, 1806) (GUIMARÃES \& D'ANDRETTA 1956: 92). Tadarida brasiliensis (I. Geoffroy, 1824) (GUIMARÃES \& D'ANDRETTA 1956: 92).

\section{Basilia dubia Guimarães \& D'Andretta, 1956}

BRASIL

Myotis nigricans (Schinz, 1821) (GUIMARÃES \& D'ANDRETTA 1956: 102). PERU

Myotis albescens (E. Geoffroy, 1806) (GUIMARÃES \& D’ANDRETTA 1956: 102).

Hospedeiro indeterminado (THEODOR 1967: 278).

VENEZUELA

Myotis albescens (E. Geoffroy, 1806)(GUIMARÃEs 1972: 3, 4; 1977: 221, 222).

Saccopteryx bilineata (Temminck, 1838) (GUIMARÃES 1972: 3).

\section{Basilia dunni Curran, 1935}

\section{PANAMÁ}

Myotis albescens (E. Geoffroy, 1806) (GUIMARÃES 1966: 399). 
Myotis nigricans (Schinz, 1821) (GUIMARÃES \& D'ANDRETTA 1956: 95; GUIMARÃES 1966: 399).

\section{VENEZUELA}

Myotis albescens (E. Geoffroy, 1806) (GUIMARÃEs 1977: 222).

\section{Basilia ferrisi Schuurmans-Stekhoven Jr., 1931}

Costa Rica

Myotis nigricans (Schinz, 1821) (GUIMARÃES \& D'ANDRETTA 1956: 75).

Myotis sp. (PETERSON 1971: 5).

Hospedeiro indeterminado (PETERSON 1971: 5).

COLÔMBIA

Myotis nigricans (Schinz, 1821) (GUIMARÃES \& D'ANDRETTA 1956: 77;

THEODOR 1967: 281).

Uroderma bilobatum Peters, 1866 (GUIMARÃES \& D'ANDRETTA 1956: 77).

Hospedeiro indeterminado (GUIMARÃEs \& D'ANDRETTA 1956: 77).

GuATEMALA

Myotis nigricans (Schinz, 1821) (GUIMARÃES \& D'ANDRETTA 1956: 76, 77).

Molossus sp. (GUIMARÃes \& D’ANDRETTA 1956: 77). GUIANA

Myotis nigricans (Schinz, 1821) (GUIMARÃES \& D'ANDRETTA 1956: 78). PANAMÁ

Myotis nigricans (Schinz, 1821) (GUIMARÃES \& D'ANDRETTA 1956: 76, 77; GUIMARÃES 1966: 399).

Hospedeiro indeterminado (GUIMARÃES \& D'ANDRETTA 1956: 77). PERU

Hospedeiro indeterminado (GUIMARÃES \& D'ANDRETTA 1956: 78). VENEZUELA

Desmodus rotundus (E. Geoffroy, 1810) (GUIMARÃES 1972: 7).

Lasiurus sp. (GUIMARÃEs \& D'ANDRETTA 1956: 78).

Molossus molossus (Pallas, 1766) (GUIMARÃEs \& D'ANDRETTA 1956: 78).

Myotis albescens (E. Geoffroy, 1806) (GUIMARÃES 1972: 7).

Myotis nigricans (Schinz, 1821) (GUIMARÃES \& D'ANDRETTA 1956: 78; GUIMARÃES 1972: 7; 1977: 222).

Myotis riparius Handley, 1960 (GUIMARÃES 1972: 7).

Myotis simus Thomas, 1901 (GUIMARÃES 1977: 222).

Myotis sp. (GUIMARÃES \& D'ANDRETTA 1956: 78).

Hospedeiro indeterminado (GUIMARÃES \& D'ANDRETTA 1956: 78).

Basilia ferruginea Miranda Ribeiro, 1903

BRASIL

Lasiurus borealis (Müller, 1776) (GUIMARÃES \& D'ANDRETTA 1956: 28). 
Hospedeiro indeterminado (GUIMARÃEs \& D'ANDRETTA 1956: 28; THEODOR 1967: 259).

CUBA

Lasiurus borealis (Müller, 1776) (GUIMARÃES \& D'ANDRETTA 1956: 28). PANAMÁ

Lasiurus borealis (Müller, 1776) (GUIMARÃES 1966: 394).

PARAGUAI

Hospedeiro indeterminado (GUIMARÃEs \& D'ANDRETTA 1956: 28; THEODOR 1967: 259).

\section{Basilia flava (Weyenbergh, 1881)}

ARGENTINA

Histiotus velatus (I. Geoffroy, 1824) (GUIMARÃES \& D'ANDRETTA 1956: 123).

\section{Basilia forcipata Ferris, 1924}

CANADÁ

Lasionycteris noctivagans (Le Conte, 1831) (WhITAKER JR. \& EASTERLA 1974: 222)

Myotis californicus (Audubon \& Bachman, 1842) (CHILTON et al. 2000: 192).

Myotis evotis (H. Allen, 1864) (PETERSON 1960: 34; CHILTON et al. 2000: 192).

Myotis lucifugus (Le Conte, 1831) (PETERSON 1960: 34; CHILTON et al. 2000: 192).

Myotis volans (H. Allen, 1866) (CHILTON et al. 2000: 192).

Myotis yumanensis (H. Allen, 1864) (PETERSON 1960: 34; 1963: 94).

Hospedeiro indeterminado (SPENCER 1937: 41).

ESTADOS UNIDOS

Euderma maculatum (J. A. Allen, 1891) (WHITAKER JR. \& EASTERLA 1975: 250).

Myotis californicus (Audubon \& Bachman, 1842) (GUIMARÃES \& D'ANDRETTA 1956: 63; PETERSON 1960: 33; 1963: 94; BRADSHAW \& ROSS 1961: 111).

Myotis evotis (H. Allen, 1864) (PETERSON 1960: 33).

Myotis lucifugus (Le Conte, 1831) (PETERSON 1960: 33; 1963: 94; HANSEN 1964: 79).

Myotis thysanodes Miller, 1897 (GUIMARÃEs \& D'ANDRETTA 1956: 63, 65; BRADSHAW \& Ross 1961: 111).

Myotis volans (H. Allen, 1866) (GUIMARÃEs \& D'ANDRETTA 1956: 63, 65; HANSEN 1964: 79; JONES JR. \& GENOWAYS 1967: 190; THEODOR 1967: 258).

Myotis yumanensis (H. Allen, 1864) (PETERSON 1960: 33; 1963: 93, 94) 
Myotis sp. (Guimarães \& D'Andretta 1956: 63; Peterson 1960: 33; 1963: 94).

Tadarida brasiliensis (I. Geoffroy, 1824) (GUIMARÃES \& D'ANDRETTA 1956: 65).

Hospedeiro indeterminado (PETERSON 1960: 33).

MÉXICO

Eptesicus fuscus (Beauvois, 1796) (WHITAKER JR. \& EASTERLA 1975: 250).

Myotis thysanodes (H. Allen, 1864) (GUIMARÃES \& D'ANDRETTA 1956: 65; WHITAKER JR. \& EASTERLA 1975: 249).

Pipistrellus hesperus (H. Allen, 1864) (GUIMARÃES \& D'ANDRETTA 1956: 65).

Tadarida brasiliensis (I. Geoffroy, 1824) (GUIMARÃES \& D'ANDRETTA 1956: 65).

\section{Basilia guimaraesi (Schuurmans-Stekhoven Jr., 1951)}

BRASIL

Myotis nigricans (Schinz, 1821) (GUIMARÃES \& D’ANDRETTA 1956: 90).

Basilia handleyi Guimarães, 1966

PANAMÁ

Lasiurus castaneus Handley, 1960 (GUIMARÃEs 1966: 396).

Basilia hughscotti Guimarães, 1946

BRASIL

Chrotopterus auritus (Peters, 1856) (GUIMARÃES \& D'ANDRETTA 1956: 116).

Basilia jellisoni Theodor \& Peterson, 1964

ESTADOS UNIDOS

Myotis yumanensis (H. Allen, 1864) (THEODOR \& PETERSON 1964: 112).

Basilia juquiensis Guimarães, 1946

BRASIL

Myotis nigricans (Schinz, 1821) (GUIMARÃES \& D'ANDRETTA 1956: 112;

GRACIOLLI \& CARVALHO 2001: 40).

Myotis sp. (GRACIOLLI \& CARVALHO 2001: 40).

VENEZUELA

Myotis riparius Handley, 1960 (GUIMARÃES 1972: 3).

Basilia lindolphoi Graciolli, 2001

BRASIL

Myotis nigricans (Schinz, 1821) (GRACIOLLI in GRACIOLLI \& CARVALHO 2001: 38). 


\section{Basilia manu Guerrero, 1996}

PERU

Myotis albescens (E. Geoffroy, 1806) (GUERRERO 1996: 668).

Myotis nigricans (Schinz, 1821) (GUIMARÃES \& D'ANDRETTA 1956: 125; GUERRERO 1996: 668).

Myotis riparius Handley, 1960 (GUERRERO 1996: 668).

Myotis simus Thomas, 1901 (GUERRERO 1996: 668).

\section{Basilia mimoni Theodor \& Peterson, 1964}

PERU

Mimon crenulatum (E. Geoffroy, 1810) (THEOdor \& PETERSON 1964: 109)

Basilia mirandariberoi Guimarães, 1942

BRASIL

Eptesicus brasiliensis (Desmarest, 1819) (GUIMARÃES \& D’ANDRETTA 1956: 111).

Myotis nigricans (Schinz, 1821) (GUIMARÃES \& D’ANDRETTA 1956: 111).

\section{Basilia neamericana Schuurmans-Stekhoven Jr., 1951}

ARGENTINA

Eptesicus diminutus Osgood, 1915 (AUTINO et al. 1999: 143)

Eptesicus furinalis (d'Orbigny, 1847) (GUIMARÃES \& D'ANDRETTA 1956: 36; AUTINo et al. 1999: 143; 2000: 110).

\section{Basilia ortizi Machado-Allison, 1963}

BRASIL

Chrotopterus auritus (Peters, 1856) (GRACIOLLI \& CARVALHO 2001: 42). CosTA RICA

Eptesicus furinalis (d'Orbigny, 1847) (PETERSON 1971: 7).

\section{VENEZUELA}

Artibeus hartii Thomas, 1892 (GUIMARÃEs 1972: 3).

Eptesicus brasiliensis (Desmarest, 1819) (MACHADO-ALLISON 1963: 458; Guimarães 1972: 3).

Eptesicus furinalis (d'Orbigny, 1847) (GUIMARÃEs 1972: 3).

Eptesicus sp. (GUIMARÃES 1977: 223).

Myotis riparius Handley, 1960 (GUIMARÃEs 1972: 3).

\section{Basilia peruvia Guimarães \& D'Andretta, 1956}

PERU

Myotis nigricans (Schinz, 1821) (GUIMARÃES \& D'ANDRETTA 1956: 84).

Tadarida brasiliensis (I. Geoffroy, 1824) (GUIMARÃES \& D'ANDRETTA 1956: 84).

Hospedeiro indeterminado (THEODOR 1967: 282). 
Basilia pizonychus Scott, 1939

MÉXICO

Myotis vivesi Menegaux, 1901 (GUIMARÃES \& D'ANDRETTA 1956: 121, 122).

\section{Basilia plaumanni Scott, 1940}

ARGENTINA

Histiotus macrotus (Poeppig, 1835) (GUIMARÃES \& D'ANDRETTA 1956: 34; CLAPS et al. 1992: 88; AUTINo et al. 1999: 145).

BRASIL

Eptesicus fuscus (Beauvois, 1796) (THEODOR 1967: 267).

Histiotus velatus (I. Geoffroy, 1824) (GUIMARÃES \& D'ANDRETTA 1956: 34).

Myotis albescens (E. Geoffroy, 1806) (AUTINO et al. 1999: 145).

Myotis nigricans (Schinz, 1821) (GUIMARÃES \& D'ANDRETTA 1956: 34).

Hospedeiro indeterminado (GUIMARÃES \& D'ANDRETTA 1956: 34).

PARAGUAI

Histiotus montanus (Philippi \& Landbeck, 1861) (GUIMARÃES \& D'ANDRETTA 1956: 34).

URUGUAI

Eptesicus brasiliensis (Desmarest, 1819) (PETERSON \& MAA 1970a: 1487).

\section{Basilia producta Maa, 1968}

BRASIL

Myotis albescens (E. Geoffroy, 1806) (MAA 1968: 30).

\section{Basilia rondanii Guimarães \& D'Andretta, 1956}

ESTADOS UNIDOS

Antrozous pallidus (Le Conte, 1856) (WHITAKER JR. \& EASTERLA 1975: 250).

Euderma maculatum (J. A. Allen, 1891)(WHITAKER JR. \& EASTERLA 1975: 250).

Myotis fortidens Miller \& Allen, 1928 (PETERSON 1963: 94).

Myotis volans (H. Allen, 1866) (WHITAKER JR. \& EASTERLA 1975: 249).

Myotis yumanensis (H. Allen, 1864) (GUIMARÃES \& D'ANDRETTA 1956: 50; WHITAKER JR. \& EASTERLA 1975: 248).

Sturnira lilium (E. Geoffroy, 1810) (PETERSON 1963: 94). GuATEMALA

Myotis nigricans (Schinz, 1821) (GUIMARÃES \& D’ANDRETTA 1956: 50). HONDURAS

Myotis nigricans (Schinz, 1821) (GUIMARÃES \& D’ANDRETTA 1956: 50). MÉXICO

Artibeus jamaicensis Leach, 1821 (GUIMARÃES \& D'ANDRETTA 1956: 50). 
Hylonycteris underwoodi Thomas, 1903 (GUIMARÃES \& D'ANDRETTA 1956: 50).

\section{Basilia rugosa Schuurmans-Stekhoven Jr., 1942}

PERU

Hospedeiro indeterminado (GUIMARÃEs \& D'ANDRETTA 1956: 124).

Basilia silvae (Brèthes, 1913)

CHILE

Histiotus velatus (I. Geoffroy, 1824) (GUIMARÃES \& D'ANDRETTA 1956: $30)$.

Myotis chiloensis (Tomes, 1857) (GUIMARÃES \& D'ANDRETTA 1956: 30; THEODOR 1967: 270).

Hospedeiro indeterminado (GUIMARÃES \& D'ANDRETTA 1956: 30).

\section{Basilia speiseri (Miranda Ribeiro, 1907)}

ARGENTINA

Phyllostoma sp. (THEODOR 1967: 283).

BRASIL

Anoura geoffroyi Gray, 1838 (GUIMARÃES \& D'ANDRETTA 1956: 71).

Carollia perspicillata (L., 1758) (GUIMARÃES \& D'ANDRETTA 1956: 71).

Eptesicus brasiliensis (Desmarest, 1819) (GUIMARÃES \& D'ANDRETTA 1956: 71).

Lasiurus borealis (Müller, 1776) (GUIMARÃEs \& D'ANDRETTA 1956: 71).

Molossus molossus (Pallas, 1766) (GUIMARÃEs \& D'ANDRETTA 1956: 72;

THEODOR 1967: 283).

Myotis nigricans (Schinz, 1821) (GUIMARÃES \& D'ANDRETTA 1956: 71).

Phyllostoma sp. (GUIMARÃES \& D'ANDRETTA 1956: 71; HURKA 1964: 77).

Hospedeiro indeterminado (GUIMARÃEs \& D'ANDRETTA 1956: 71, 72).

PARAGUAI

Eptesicus furinalis (d'Orbigny, 1847) (GUIMARÃES \& D'ANDRETTA 1956: 72).

Myotis albescens (E. Geoffroy, 1806) (GUIMARÃES \& D'ANDRETTA 1956: 72).

Hospedeiro indeterminado (GUIMARÃES \& D'ANDRETTA 1956: 72).

LOCALIDADE INDETERMINADA

Molossus molossus (Pallas, 1766) (GUIMARÃEs \& D'ANDRETTA 1956: 72).

\section{Basilia tiptoni Guimarães, 1966}

BRASIL

Mimon crenulatum (E. Geoffroy, 1810) (KOMENO \& LINHARES 1999: 154). PANAMÁ

Lonchorhina sp. ou Tonatia sp. (GUIMARÃES 1966: 398).

Mimon crenulatum (E. Geoffroy, 1810) (GUIMARÃES 1966: 398). 
VENEZUELA

Mimon crenulatum (E. Geoffroy, 1810) (GUIMARÃES 1972: 2).

Basilia traubi Maa, 1968

MÉXICO

Myotis sp. (MAA 1968: 28).

\section{Basilia travassosi Guimarães, 1938}

BRASIL

Myotis albescens (E. Geoffroy, 1806) (GUIMARÃEs \& D'ANDRETTA 1956: 89).

Hospedeiro indeterminado (GUIMARÃES \& D'ANDRETTA 1956: 89).

\section{Basilia tuttlei Guimarães, 1972}

VENEZUELA

Myotis nigricans (Schinz, 1821) (GUIMARÃES 1972: 5).

\section{Basilia typhlops Guimarães, 1972}

VENEZUELA

Myotis oxyotus (Peters, 1867) (GUIMARÃes 1972: 6).

\section{Basilia wenzeli Guimarães \& D'Andretta, 1956}

COlÔMBIA

Histiotus sp. (GUIMARÃES \& D’ANDRETTA 1956: 42).

PANAMÁ

Artibeus jamaicensis Leach, 1821 (GUIMARÃES 1966: 396).

Eptesicus brasiliensis (Desmarest, 1819) (GUIMARÃES 1966: 396).

VENEZUELA

Eptesicus furinalis (d'Orbigny, 1847) (GUIMARÃES 1972: 2).

Eptesicus fuscus (Beauvois, 1796) (GUIMARÃES \& D'ANDRETTA 1956: 42).

Lonchorhina aurita Tomes, 1863 (GUIMARÃES \& D'ANDRETTA 1956: 42).

Hospedeiro indeterminado (GUIMARÃEs \& D'ANDRETTA 1956: 42; HURKA 1964: 77).

\section{Basilia sp.}

ARGENTINA

Eptesicus furinalis (d'Orbigny, 1847) (AUTINo et al. 1999: 146).

Myotis albescens (E. Geoffroy, 1806) (AUTINO et al. 1999: 146).

Myotis riparius Handley, 1960 (AUTINO et al. 1999: 146).

BRASIL

Myotis riparius Handley, 1960 (WHITAKER JR. \& MUMFORD 1977: 257). CANADÁ

Eumops perotis (Schinz, 1821) (SPENCER 1939: 17). 
COLÔMBIA

Eptesicus fuscus (Beauvois, 1796) (MARINKELLE \& GROSE 1981: 13)

Myotis nigricans (Schinz, 1821) (MARINKELLE \& GROSE 1981: 13)

Platyrhinus vittatus (Peters, 1860) (MARINKELLE \& GROSE 1981: 13)

EL SALVADOR

Myotis nigricans (Schinz, 1821) (STARRET \& DE LA TORRE 1964: 62)

VENEZUELA

Mimon crenulatum (E. Geoffroy, 1810) (GUIMARÃES 1972: 7).

Myotis nigricans (Schinz, 1821) (GUIMARÃEs 1972: 7).

Myotis riparius Handley, 1960 (GUIMARÃEs 1972: 7).

Basilia sp. A

COLÔMBIA

Myotis nigricans (Schinz, 1821) (GUIMARÃES \& D'ANDRETTA 1956: 124).

Basilia sp. C

Costa RicA

Centurio senax Gray, 1842 (GuIMARÃES \& D'ANDRETTA 1956: 125).

Eptesicus sp. (PETERSON 1971: 7).

Basilia sp. D

ESTADOS UNIDOS

Plecotus rafinesquii Lesson, 1827 (GUIMARÃEs \& D’ANDRETTA 1956: 126).

Basilia sp. E

Bolívia

Myotis nigricans (Schinz, 1821) (GUIMARÃES \& D'ANDRETTA 1956: 126).

Hershkovitzia Guimarães \& D'Andretta, 1956

Hershkovitzia cabala Peterson \& Lacey, 1985

BRASIL

Thyroptera tricolor Spix, 1823 (PETERSON \& LACEY 1985: 580).

Hershkovitzia coeca Theodor, 1967

LOCALIDADE INDETERMINADA

Hospedeiro indeterminado (THEODOR 1967: 348).

Hershkovitzia inaequalis Theodor, 1967

PERU

Thyroptera discifera (Lichtenstein \& Peters, 1855) (THEODOR 1967: 352).

Hershkovitzia primitiva (Guimarães \& D'Andretta, 1956)

COLÔMBIA

Thyroptera discifera (Lichtenstein \& Peters, 1855) (GUIMARÃES \& D'ANDRETTA 1956: 130). 
Tabela I. Quadro sinóptico da ocorrência das espécies americanas de Nycteribiidae.

\begin{tabular}{ll}
\hline \multicolumn{1}{c}{ Pais } & \multicolumn{1}{c}{ Espécie de Nycteribiidae } \\
\hline $\begin{array}{l}\text { Argentina } \\
\text { Bolivia }\end{array}$ & Basilia carteri, B. currani, B. flava, B. neamericana, B. plaumanni, B. speiseri \\
Brasil & Basilia carteri \\
& Basilia andersoni, B. carteri, B. currani, B. dubia, B. ferruginea, B. guimaraesi, B. hughscotti, B. \\
& juquiensis, B. lindolphoi, B. mirandariberoi, B. ortizi, B. plaumanni, B. producta, B. speiseri, B. \\
Canadá & Bastoni, B. travassosi, Hershkovitzia cabala \\
Chile & Basilia silvae \\
Colömbia & Basilia anceps, B. astochia, B. bequaerti, B. constricta, B. ferrisi, B. wenzeli, Hershkovitzia primitiva \\
Costa Rica & Basilia costaricensis, B. ferrisi, B. ortizi \\
Cuba & Basilia cubana, B. ferruginea \\
Equador & Basilia constricta \\
Estados Unidos & Basilia antrozoi, B. boardmani, B. corynorhini, B. forcipata, B. jellisoni, B. rondanii \\
Guatemala & Basilia anomala, B. ferrisi, B. rondanii \\
Guiana & Basilia ferrisi \\
Honduras & Basilia rondanii \\
México & Basilia anomala, Basilia antrozoi, B. bellardii, B. forcipata, B. pizonychus, B. rondanii, B. traubi \\
Nicarágua & Basilia anomala \\
Panamá & Basilia dunni, B. ferrisi, B. ferruginea, B. handleyi, B. tiptoni, B. wenzeli \\
Paraguai & Basilia bequaerti, Basilia carteri, B. ferruginea, B. plaumanni, B. rugosa, B. speiseri \\
Peru & Basilia anceps, B. constricta, B. dubia, B. ferrisi, B. manu, B. mimoni, B. peruvia, Hershkovitzia \\
inaequalis & Basilia andersoni, Basilia carteri, B. plaumanni \\
Uruguai & Basilia anceps, B. anomala, B. bequaerti, B. constricta, B. dubia, B. dunni, B. ferrisi, B. juquiensis, \\
Venezuela & B. ortizi, B. tiptoni, B. tuttlei, B. typhlops, B. wenzeli \\
\hline
\end{tabular}

\section{REFERÊNCIAS BIBLIOGRÁFICAS}

Autino, A.G.; G.L. ClıPS \& R.M. BARQuez. 1999. Insectos ectoparasitos de murcielagos de las Yungas de la Argentina. Acta Zool. Mex., n.s., 78: 119-169.

- 2000. Nuevos registros de Diptera (Nycteribiidae) y Siphonaptera (Ischnopsyllidae) de Chiroptera (Vespertilionidae) de la Argentina. Bol. Entomol. Venez. 15: 109-112.

Bradshaw, G.V.R. \& A. Ross. 1961. Ectoparasites of Arizona bats. Jour. Ariz. Acad. Sci. 1: 109-112.

Chilton, G.; M.J. Vonhof; B.V. Peterson; N. Wilson. 2000. Ectoparasitic insects of bats in British

Columbia, Canada. Jour. Parasitol. 86: 191-192.

Claps, G.L.; A.G. Autino \& R.M. Barquez. 1992. Nuevas citas de dipteros ectoparasitos (Nycteribiidae) para murcielagos de la Argentina. Rev. Soc. Entomol. Argent. 50: 88.

Claps, G.L.; A.G. Autino \& A.M. Saralegui. 1998.Insectos ectoparásitos de dos especies de Myotis

(Chiroptera: Vespertilionidae) del Uruguay. Neotrópica 44: 113-114.

Graciolli, G. \& C.J.B. de Carvalho. 2001. Moscas ectoparasitas (Diptera, Hippoboscoidea, Nycteribiidae) de morcegos (Mammalia, Chiroptera) do Estado do Paraná, Brasil. I. Basilia, chave pictórica para as espécies e taxonomia. Revta bras. Zool. 18 (Supl. 1): 33-49.

Guerrero, R. 1996. The Basilia juquiensis species-group (Diptera: Nycteribiidae) with description of a new species from Pakitza, Peru, p. 665-674. In: D.E. Wilson \& A. SANDOval (Eds). Manu: La biodiversidad del Sureste del Peru. Washington, D.C., Smithsonian Institution, 679p.

GuimARÃES, L.R. 1966. Nycteribiid batflies from Panama (Diptera: Nycteribiidae), p. 393-404. In: R.L.

WenZel \& V.J. TiPTON (Eds). Ectoparasites of Panama. Chicago, Field Mus. Nat. Hist., XII+861p. 1972. Venezuelan nycteribiid batflies (Diptera:Nycteribiidae). Brigham Young Univ. Sci.

Bull., Biol. Ser., 17: 1-10.

- 1977. Supplementary note on Venezuela bat flies (Diptera: Nycteribiidae). Gt Basin Nat. 37: 221-224.

Guimarães, L.R. \& M.A.V. D'Andretta. 1956. Sinopse dos Nycteribiidae (Diptera) do Novo Mundo.

Arq. Zool. São Paulo 9: 1-175.

HANSEN, C.G. 1964. Ectoparasites of mammals from Oregon. Gt Basin Nat. 24: 75-81. 
Hobson, C.S. 2000. First record of Nycteribiidae (Diptera) in Virginia, and a discussion of the host specificity and distribution of Basilia boardmani. Ent. News 111: 291-293.

HURKA, K. 1964. Revision der Nycteribiidae und Streblidae-Nycteriboscinae aus der dipterensammlung des Zoologischen Museums in Berlin. Mitt. Zool. Mus. Berlin 40: 71-86.

- 1970. Basilia (Basilia) cubana sp. n., a new bat fly from Cuba (Diptera, Nycteribiidae). Acta ent. bohemolov. 67: 335-338.

Komeno, C.A. \& A.X. Linhares. 1999. Batflies parasitic on some phyllostomid bats in Southeastern Brazil: parasitism rates and host-parasite relationships. Mem. Inst. Oswaldo Cruz 94: 151-156.

Koopman, K.F. 1993. Order Chiroptera, p. 137-241. In: D.L. Wilson \& D.M. ReEder (Eds). Mammals species of the world. A taxonomic and geographic reference. Washington, D.C., Smithsonian Institution Press, $2^{\text {nd }}$ ed., 1206p.

JoNES JR., J.K. \& H.H. GenowaYs. 1967. Annotated checklist of bats from South Dakota. Trans. Kansas Acad. Sci. 70: 184- 196.

LEWIS, S.E. 1996. Low roost-site fidelity in pallid bats: associated factors and effect on group stability. Behav. Ecol. Sociobiol. 39: 335-344.

MAA, T.S. 1968. New Basilia species from Thailand, Mexico and Brazil (Diptera: Nycteribiidae). Pacif. Insects 10: 25-32.

Machado-Allison, C.E. 1963. Un nuevo Nycteribiidae (Diptera) de Venezuela. Acta Biol. Ven. 23: 455-459.

Marinkelle, C.J. \& E.S. Grose. 1981. A list of ectoparasites of Colombian bats. Rev. Biol. Trop. 29: 11-20.

Peterson, B.V. 1960. New distribution and host records for bat flies, and a key to the north american species of Basilia Ribeiro (Diptera: Nycteribiidae). Proc. Ent. Soc. Ont. 90: 30-36.

- 1963. Additional records of some american bat flies (Diptera: Nycteribiidae). Proc. Ent. Soc. Ont. 93: 93-94.

1971. Notes on the bat flies of Costa Rica (Diptera: Nycteribiidae). Contr. Sci. 212: 1-8.

Peterson, B.V. \& L.A. LACEY. 1985. A new species of Hershkovitzia (Diptera: Nycteribiidae) from Brazil, with a key to the described species of the genus. Proc. Entomol. Soc. Wash. 87: 578-582.

PETERSON, B.V. \& T.C. MAA. 1970a. One new and one previously unrecorded species of Basilia (Diptera: Nycteribiidae) from Uruguay. Can. Ent. 102: 1480-1487.

- 1970b. A new species of Basilia (Diptera: Nycteribiidae) from Colombia. Can. Ent. 102: 1519-1523.

RICE, D.W. 1957. Life history and Ecology of Myotis austroriparius in Florida. Jour. Mammal. 38: 15-32.

RICHERSON, J.V.; J.F. SCUDDAY \& S.B. TABOR. 1992. An ectoparasite survey of mammals in Brewster County, Texas, 1982-1985. Southwest. Entomol. 17: 7-15.

SPENCER, G.J. 1937. Ectoparasites of birds and mammals of British Columbia. II. A preliminary list of the Pupipara, louse flies. Proc. Ent. Soc. Brit. Columbia 34: 39-42.

. 1939. Ectoparasites of birds and mammals of British Columbia. IV: The parasite of bats. Proc. Ent. Soc. Brit. Columbia 36: 16-18.

StARRET, A. \& L. DE LA TORRE. 1964. Notes on a collection of bats from Central America, with the third record for Cyttarops alecto Thomas. Zoologica 49: 53-63.

THEODOR, O. 1967. An illustrated catlogue of the Rothschild Collection of Nycteribiidae (Diptera) in the British Museum (Natural History) with keys and short descriptions for the identification of subfamilies, genera, species and subspecies. British Museum (Natural History) Publication 665: $1-506$.

Theodor, O. \& B.V. Peterson. 1964. On some new species of Nycteribiidae (Diptera: Pupipara). Gt Basin Nat. 24: 107-115.

Wenzel, R.L.; V.J. Tipton \& A. KIEWLicz. 1966.The streblid batflies of Panama (Diptera: Calyptera: Streblidae), p. 405-675. In: R.L. WENZEL \& V.J. TIPTON (Eds). Ectoparasites of Panama. Field Mus. Nat. Hist., Chicago, XII+861p. 
WhITAKER JR., J.O. \& D.A. EASTERLA. 1974. Batflies (Streblidae and Nycteribiidae) in the eastern United States, and a nycteribiid record from Saskatchewan. Ent. News 85: 221-223. 1975. Ectoparasites of bats from Big Bend National Park, Texas. Southwest. Nat. 20: 241-254.

WhitAKER JR., J. \& R.E. MUMFoRD. 1977. Records of ectoparasites from brazilian mammals. Ent. News 88: $255-258$.

Recebido em 29.VI.2000; aceito em 02.VII.2001. 